Western University

Scholarship@Western

Psychology

Psychology

2005

\title{
Intelligence correlates of transcendent beliefs: A preliminary study
}

Nicole Lukey

Imants Barušs

King's University College, baruss@uwo.ca

Follow this and additional works at: https://ir.lib.uwo.ca/kingspsychologypub

Part of the Psychology Commons

Citation of this paper:

Lukey, Nicole and Barušs, Imants, "Intelligence correlates of transcendent beliefs: A preliminary study" (2005). Psychology. 2.

https://ir.lib.uwo.ca/kingspsychologypub/2 
Intelligence Correlates of Transcendent Beliefs 1

Intelligence Correlates of Transcendent Beliefs: A Preliminary Study

Nicole Lukey and Imants Barušs

Department of Psychology

King's University College at The University of Western Ontario

(C) 2004 Nicole Lukey and Imants Barušs 
Intelligence Correlates of Transcendent Beliefs 2

\begin{abstract}
In previous research Barušs and Moore had identified a material-transcendent dimension of beliefs about consciousness and reality that underlies the Western intellectual tradition including the academic study of consciousness. At one pole, materialists believe that reality is entirely physical in nature, whereas those tending toward the transcendent pole believe that reality cannot exhaustively be captured in physical terms. More recently Jewkes and Barušs had found a number of personality correlates of transcendent beliefs including a tendency toward curiosity and a rational approach to the world. These previous results prompted the present study in which 39 undergraduate psychology students at a liberal arts college were given a measure of beliefs about consciousness and reality, a comprehensive intelligence test, and a personality subtest. Correlations were found among various facets of intelligence, aspects of beliefs, and intellectual curiosity. The results indicate that greater intelligence is associated with transcendent beliefs.
\end{abstract}


Intelligence Correlates of Transcendent Beliefs 3

\section{Introduction}

In 1983 Imants Barušs and Robert Moore were trying to advance the psychological study of consciousness when they noticed that there was considerable disparity in the way that consciousness was conceptualized by scientists, academics, and mental health professionals who were conducting research concerning consciousness. In fact, ideas about consciousness were sometimes diametrically opposed to one another. For example, some researchers said that consciousness was entirely a byproduct of physiological processes, whereas others maintained that it was not. It appeared that these ideas about consciousness, rather than reflecting objective scientific investigation, were tied to investigators' personal beliefs about the nature of reality. In particular, there appeared to be a material-transcendent dimension of beliefs about reality underlying the discussion of consciousness in the academic literature. Barušs and Moore decided to conduct a survey to investigate the apparent link between notions of consciousness and personal beliefs about the nature of reality (Barušs, 1990).

The study by Barušs and Moore consisted of the development of questionnaires to measure ideas about consciousness and beliefs about reality and their administration in preliminary, pilot, and survey stages of the research. The final survey, in 1986, of academics and professionals who could potentially write about consciousness in the academic literature, yielded 334 completed questionnaires. Extensive multivariate statistical analyses, including hierarchical cluster analyses and principal components factor analyses with varimax rotations, revealed substantial correlations between ideas about consciousness and beliefs about reality. In fact, a material-transcendent dimension could be identified with materialist, conservatively transcendent, and extraordinarily transcendent positions. Those tending toward the materialist pole believe that reality is entirely physical in nature, that science is the proper way to study it, and that consciousness is an emergent property of the brain or sufficiently complex information-processing systems, or at any rate, that consciousness is just more information in an information-processing system. Those tending toward the conservatively transcendent position believe that there is more to reality than the physical universe; that science is not always the best way to know something; and that consciousness is fundamentally subjective in nature, gives meaning to reality, and is evidence of a spiritual dimension within a person. Those tending toward the extraordinarily transcendent pole of the materialtranscendent dimension believe that physical reality is an extension of mental reality, that there are ways of knowing that are superior to rational thought, and that there is a universal consciousness underlying all of reality of which we are a part and which we can come to know through a process of self-transformation (Barušs, 1990). Furthermore, it is clear from these analyses that the importance of consciousness increases from being unimportant for the materialist, to being important for those identified with the conservatively transcendent position, to being all that exists for those with extraordinarily transcendent beliefs (Barušs, 2001).

Given the strong associations found between notions of consciousness and beliefs about reality, the distinction between the two was dropped, and they were subsequently collectively referred to simply as beliefs about consciousness and reality. Thirty-eight items making up six factors of the final 
Intelligence Correlates of Transcendent Beliefs 4

survey results, were redefined as the Beliefs About Consciousness and Reality Questionnaire (BACARQ, Barušs \& Moore, 1992).

The structure of the BACARQ consists of a global scale called Global Beliefs that includes all 38 questionnaire items and six subscales, made up of 7 to 12 items each, with the global scale and all subscales oriented in the transcendent direction for convenience. The Global Beliefs Scale is a measure of a person's position on the material-transcendent dimension. A person's belief that reality is not entirely physical is gauged by the Antiphysicalism subscale. The Meaning subscale reflects not only the belief that there is more to reality than that which is physical, but also that meaning and spirituality have to be taken into account. Explicitly religious beliefs, such as conviction in the existence of an original creator or the existence of absolute truth, are captured by the Religiosity subscale. The Extraordinary Experiences subscale reflects the degree to which a person believes that she has had unusual experiences such as transcendent or out-of-body experiences. The Extraordinary Beliefs subscale measures unusual beliefs such as belief in the existence of reincarnation or the existence of a universal consciousness. Finally, a person's belief that it is necessary to change oneself through inner examination and some form of practice such as meditation are gauged by the Inner Growth subscale. The subscale reliabilities range from a Cronbach's alpha value of .82 to an alpha of .89 with the global scale having an overall alpha of .95 , thereby indicating that the scales are measuring coherent psychological constructs (Barušs \& Moore, 1992; 1998).

Since its inception, the BACARQ has been used in a number of studies. About 1000 copies were handed to each of the participants at the "Toward a Science of Consciousness 1996 "Tucson II" academic conference, and the scores obtained from 212 completed questionnaires were compared to those of the original 1986 sample (Barušs and Moore, 1998). Students have been given the BACARQ at the beginning, at the half-way point, and at the ends of courses taught by different professors with the finding that students sometimes appear to shift their stated beliefs in the direction of their instructors' beliefs (Barušs, 2000). Most significantly perhaps, it was found with a student sample that those tending toward transcendent beliefs are more curious and have a more rational approach to the world than those with more materialist beliefs (Jewkes \& Barušs, 2000).

The relationship between transcendence and rationality was found in a study in which 75 participants were given Jackson's Personality Research Form E (PRF-E, 1999) as well as the BACARQ, and a statistically significant Pearson product moment correlation coefficient of .57 was found between the Understanding Scale, which is a measure of intellectual curiosity and rationality, and the Global Beliefs Scale of the BACARQ. In fact, there were statistically significant correlations between the Understanding Scale and all of the BACARQ subscales ranging in value from .32 to .61. It is unlikely that these results can be attributed to efforts on the part of more conscientious students to please the investigators since scores on the control Desirability Scale were uncorrelated with the BACARQ scales and since there were statistically significant negative correlations between the measured need for social recognition and transcendence. In other words, students who were not only more curious but also more independent were more likely to have transcendent beliefs (Jewkes \& Barušs, 2000). 
The Understanding Scale of the PRF-E is related to the personality construct of openness which is known to have small positive correlations with intelligence as measured by standard intelligence tests (e.g., McCrae, 1993-94). But because understanding is correlated with transcendent beliefs, the question naturally arises of whether intelligence itself is correlated with transcendent beliefs.

As can be seen from a description of the BACARQ subscales, transcendent beliefs have a multifaceted structure that includes belief in the occurrence of unusual phenomena. However, within the scientific community, such beliefs are thought to be associated with irrationality and a lack of critical judgment. Or to put it bluntly, there appears to be a belief among some scientists that those who think that there is more to reality than the physical universe are just plain stupid and that if they had more wits they would be materialists. Some support for such a contention has been found in studies such as that of Tobacyk and Milford (1983) in which there were some correlations between some paranormal beliefs, such as beliefs in witchcraft, spiritualism, and superstitions, and some measures of irrational and uncritical thinking. Overall, however, the evidence for a cognitive deficits hypothesis of paranormal beliefs has been inconsistent and inconclusive (Roe, 1999).

In an actual test of critical thinking, Roe had 117 undergraduate university students evaluate fictional experimental reports about the existence of ESP. Some of the reports were pro-ESP and some were anti-ESP but all had a number of methodological and analytical flaws. There were no differences in the critical evaluation of the reports by believers and disbelievers of the paranormal. However, participants who were asked to evaluate a report that reached a conclusion contrary to their own a prior beliefs gave a lower rating to the report than those whose beliefs were congruent with the conclusion of the paper (Roe, 1999).

While the cognitive deficits hypothesis literature is relevant to the present study, the BACARQ was not designed to measure paranormal beliefs as such, but rather transcendent beliefs. For example, there are no items on the BACARQ to assess superstitious behaviours such as beliefs that black cats are unlucky or that the number 13 is unlucky (cf. Tobacyk and Milford, 1983). Items on the BACARQ that do consist of statements about paranormal phenomena, such as the statement that reincarnation exists, could be endorsed, on the face of it, either without any critical reflection or after considerable critical reflection. Given the equivocal nature of its results and only partial overlap with the present study, it is hypothesized that the cognitive deficits hypothesis, to the extent that it can be tested, will not be borne out in this study.

The purpose of this study is to establish the relationship between intelligence and beliefs about consciousness and reality. In addition, the relationships of both intelligence and beliefs to the personality trait of understanding will be explored. In light of the absence of any conclusive associations of aspects of transcendence with lack of critical thinking and, on the contrary, the presence of previously found correlations between understanding and transcendence, we hypothesize that correlations will be found between intelligence as measured by an intelligence test and transcendence as measured by the BACARQ. In addition, positive correlations will be found between understanding, as measured by the Understanding Scale of the PRF-E, and both intelligence and transcendent beliefs. 
Intelligence Correlates of Transcendent Beliefs 6

\section{Method}

This study was conducted in the Psychology Laboratory at King's University College, a Catholic liberal arts college affiliated with the University of Western Ontario. Participants were recruited through a course web site and posters and consisted of 39 undergraduate psychology students, 9 male and 30 female, who earned up to $5 \%$ of their final grade in introductory psychology by completing a short assignment based on their participation in the study. The mean age of all participants was 20.2 years with a standard deviation of 1.6 and range in age from 19 to 25 years. Thirty-two participants were in their first year of university, 5 in their second year, and 2 in their third year. Twenty-six participants were Christian, 10 indicated that they had their own beliefs, and 3 fell into other categories. Thirteen participants indicated that they never practised their religious beliefs, 6 practised annually, 9 monthly, 7 weekly, and 4 daily.

Jackson's Multidimensional Aptitude Battery-II (MAB-II, 1998) was used to assess the intelligence of participants. The MAB-II is an intelligence test with five subtests used to measure Verbal IQ and five subtests used to measure Performance IQ. The Verbal and Performance components of the test are combined to calculate a Full Scale IQ, a measure of a person's intelligence. The MAB-II has testretest reliability values of .95 for the Verbal Scale, .96 for the Performance Scale, and .97 for the Full Scale. Due to time constraints, only 6 of the possible 10 subtests were administered, three verbal and three performance, namely, Information, Arithmetic, Vocabulary, Digit Symbol, Spatial, and Object Assembly.

Participants' beliefs were assessed using Barušs and Moore's Beliefs About Consciousness and Reality Questionnaire. The BACARQ contains 38 statements about consciousness and reality to which respondents indicate their agreement on four-point and seven-point Likert-type scales. Responses are scored zero for the median choice with negative and positive values used for choices below and above the median respectively. Analysis in the past of the reliability of the BACARQ using student data has given values for Cronbach's alpha between .88 and .92 on the Global Beliefs Scale (Barušs, 2000).

The personality trait of understanding was measured using the Understanding Scale from Jackson's Personality Research Form E. This test consists of 16 statements to which respondents answer with "true" or "false." The Understanding Scale has an odd-even reliability of .77 and test-retest reliability values of .89 and .93 for previous forms (Jackson, 1999).

Demographic information was collected by way of a Demographic Information Questionnaire containing items regarding a participant's age, sex, highest level of education, religious affiliation, frequency of religious or spiritual practice, and whether or not she was part of the psychology research participant pool.

Participants entered one of the Psychology Laboratory rooms at King's College in small groups and sat down at tables. Written informed consent was obtained after they had had an opportunity to read a one paragraph description of the study. Participants used either a pen or a pencil to answer the 
Intelligence Correlates of Transcendent Beliefs 7

Demographic Information Questionnaire, the Understanding Scale of the PRF-E, the BACARQ, and the MAB-II. During the Arithmetic test of the MAB-II, each participant was given one $81 / 2$ " by $11 "$ piece of paper. A stopwatch was used to time each of the sections of the MAB-II. Finally, participants were debriefed and allowed time to complete research assignments based on their participation in the study.

\section{Results}

The purpose of the study was to examine the relationship between intelligence and beliefs about consciousness and reality. The Full Scale IQ scores on the intelligence test range from 82 to 127 with a mean of 107 and standard deviation of 9 . The average is slightly above the norm as could be expected of university students. All subscale IQ scores used in analyses were age-adjusted. Global Beliefs scores on the BACARQ range from -19 to 70 with a mean of 21 and standard deviation of 19. This average is comparable to previous average scores for students that have ranged from 14 to 46 with standard deviations having values of 15 to 34 (Barušs, 2000; Jewkes \& Barušs, 2000). By way of further comparison, the average score of 212 respondents at the "Toward a Science of Consciousness 1996 'Tucson II'" academic meeting was 18 with a standard deviation of 41 (Barušs $\&$ Moore, 1998). The scores for the Understanding Scale range from 3 to 15 with a mean of 8 and standard deviation of 3 . In a previous study with a student sample the average was 9 with a standard deviation of 3 (Jewkes \& Barušs, 2000). All of the scale scores are reasonably normally distributed.

The internal consistencies of the global scale and subscales of the BACARQ were checked by calculating Cronbach's alpha for each of the scales. The alpha value of the global scale is .79. The values for the subscales range from.50 to .71 for all of the subscales except for Meaning which has a value of .28. Such a low value of Cronbach's alpha means that the items making up the scale are not forming a single construct. At least three ideas are represented by the items of this scale: the importance of meaning, the extension of reality beyond the physical universe, and the presence of a spiritual reality. With the previous samples of academics and professionals, this scale had alpha values of .84 and .77 (Barušs \& Moore, 1992; 1998) indicating an underlying psychological construct that could be expressed as the belief that meaning is a spiritual reality that cannot be accounted for entirely in physical terms. Given the previously found coherence of the scale and its conceptual meaningfulness, it was decided to use the scale without modification.

The main statistical analyses used for interpreting the data were correlational. All of the correlations statistically significant at the $p<.05$ level between all IQ scales and subscales and all BACARQ scales and subscales are in the expected direction; that is to say, all of the statistically significant correlations are positive, as can be seen in Table 1. There is no statistically significant overall correlation at the $p<.05$ level of statistical significance between Full Scale IQ and global transcendent beliefs. However, there is a statistically significant correlation between Full Scale IQ and the Meaning subscale of the BACARQ. There are also correlations between Verbal IQ and the Meaning and Extraordinary Experiences subscales of the BACARQ. One of the Verbal subscales, Vocabulary, is correlated with Inner Growth, and one of the Performance subscales, Object Assembly, is correlated with both the Antiphysicalism and Meaning subscales of the BACARQ. In 
Intelligence Correlates of Transcendent Beliefs 8

addition, there is a suggestive correlation between Verbal IQ and Global Beliefs $(r=.29, p=.08)$. All tests of statistical significance in these results are two-tailed.

TABLE 1

Correlations of IQ With Beliefs Scales

\begin{tabular}{|l|l|c|c|c|c|}
\hline & & Antiphysicalism & Meaning & $\begin{array}{c}\text { Extraordinary } \\
\text { Experiences }\end{array}$ & Inner Growth \\
\hline \multirow{4}{*}{ IQ } & Vocabulary & & & & $\mathbf{4 1}$ \\
\cline { 2 - 6 } & $\begin{array}{l}\text { Object } \\
\text { Assembly }\end{array}$ & .37 & .35 & & \\
\cline { 2 - 6 } & & .33 & .33 & \\
\cline { 2 - 6 } & Verbal & & .33 & & \\
\cline { 2 - 6 } & Full Scale & & & & \\
\hline
\end{tabular}

Table 1 Note: Correlations in bold are statistically significant at $p<.01$ and all others at $p<.05$.

The strongest correlations of IQ scales and subscales with individual items of the BACARQ are with Item Q09, which is a key item that reads: "There is no reality other than the physical universe." The correlations that are at least marginally statistically significant are given in Table 2. In some sense, this item defines the core construct of the material-transcendent beliefs dimension. Its strong negative correlations with a broad spectrum of facets of intelligence indicate that those who are more intelligent are less likely to think that the physical universe is all there is to reality. 
Intelligence Correlates of Transcendent Beliefs 9

TABLE 2

Correlations of IQ With Individual Beliefs Items

\begin{tabular}{|c|l|c|c|}
\hline & & Q09 & $p$ \\
\hline \multirow{7}{*}{ IQ } & Information & -.44 & .005 \\
\cline { 2 - 4 } & Arithmetic & -.34 & .034 \\
\cline { 2 - 4 } & Vocabulary & -.45 & .004 \\
\cline { 2 - 4 } & Object Assembly & -.40 & .011 \\
\cline { 2 - 4 } & Verbal & -.52 & .001 \\
\cline { 2 - 4 } & Performance & -.29 & .074 \\
\cline { 2 - 4 } & Full Scale & -.48 & .002 \\
\hline
\end{tabular}

Correlations of the Understanding Scale of the PRF-E with IQ scales and subscales and BACARQ scales and subscales were calculated. Those that are at least marginally statistically significant are given in Table 3. There are statistically significant correlations of Understanding with Verbal IQ of the MAB-II as well as with the Global Beliefs Scale of the BACARQ.

TABLE 3

Correlations of Understanding With IQ and Beliefs

\begin{tabular}{|l|l|c|c|}
\hline \multirow{4}{*}{ IQ } & & Understanding & $p$ \\
\hline \multirow{4}{*}{ Beliefs } & Information & .27 & .099 \\
\cline { 2 - 4 } & Vocabulary & .49 & .002 \\
\cline { 2 - 4 } & Verbal & .35 & .029 \\
\hline \multirow{5}{*}{} & Religiosity & .29 & .074 \\
\cline { 2 - 4 } & Meaning & .29 & .072 \\
\cline { 2 - 4 } & Extraordinary & .28 & .085 \\
\cline { 2 - 4 } & Experiences & & .001 \\
\cline { 2 - 4 } & Inner Growth & .49 & .029 \\
\cline { 2 - 4 } & Global Beliefs & .35 & \\
\hline
\end{tabular}

It should be noted that all of the preceding analyses were done with the assumption that the relationships between all of the variables are linear. However, examination of the scatterplots of 
some of the data for the global scales suggest fitting them with quadratic equations. Indeed, when the Global Beliefs scores are treated as the dependent variable and Full Scale IQ as the independent variable, a quadratic equation gives a better fit than a linear equation. In particular, those with lower Full Scale IQ as well as those with higher Full Scale IQ had higher values of Global Beliefs than those with midrange Full Scale IQ scores. Similar results carry through also when Verbal and Performance scales scores are treated as the independent variables for quadratic equations in which Global Beliefs are the dependent variable. The improvement in goodness of fit can be seen by inspecting the $R^{2}$ values given in Table 4 .

TABLE 4

Values of $R^{2}$ for Global Beliefs Regressed on IQ

\begin{tabular}{|l|c|c|c|}
\hline \multirow{2}{*}{ Relationship } & \multicolumn{3}{|c|}{ IQ } \\
\cline { 2 - 4 } & Verbal & Performance & Full Scale \\
\hline Linear & .082 & .015 & .050 \\
\hline Quadratic & .101 & .101 & .115 \\
\hline
\end{tabular}

Another way of noting the difference between linear and quadratic relationships between Global Beliefs and IQ scales is to use trend analysis. The IQ scores were split into 6 categories at $1 / 2 \sigma$ intervals away from the mean. In the case of Performance IQ, whereas there was no linear trend, a quadratic trend was present $(p<.05)$. However, this was not the case for Verbal IQ or Full Scale IQ.

The most dramatic improvement from modelling the data using quadratic equations rather than linear equations occurs for the relationship between Performance IQ and Global Beliefs. Another way of conceptualizing this relationship is to split Performance IQ at the median and to consider the linear correlations for the lower and upper halves of the data. Such post hoc analyses do not result in any statistically significant correlations below the median but they do for correlations above the median as shown in Table 5. Hence, Performance IQ is correlated with transcendent beliefs, but only when Performance IQ values are sufficiently large. The same post-hoc procedures do not yield any substantially new insights in the case of Verbal and Full Scale IQ relationships with Global Beliefs. 
Intelligence Correlates of Transcendent Beliefs 11

TABLE 5

Correlations of Performance IQ Above the Median With Beliefs Scales

\begin{tabular}{|l|c|c|}
\hline & Performance IQ & $p$ \\
\hline Antiphysicalism & .49 & .041 \\
\hline Religiosity & .42 & .080 \\
\hline Meaning & .63 & .005 \\
\hline $\begin{array}{l}\text { Extraordinary } \\
\text { Experiences }\end{array}$ & .54 & .020 \\
\hline Extraordinary Beliefs & .46 & .057 \\
\hline Inner Growth & .40 & .102 \\
\hline Global Beliefs & .65 & .003 \\
\hline
\end{tabular}

Table 5 Note: These correlations are based on the 18 cases that fall above the median value of 107 for Performance IQ.

\section{Discussion}

The hypotheses for this study, that intelligence is correlated with transcendent beliefs, and that both are correlated with the personality trait of understanding, are, in large part, borne out. Even though the overall correlation $(r=.22)$ between Full Scale IQ and the Global Beliefs Scale was not statistically significant, there were statistically significant correlations between some IQ subscales and Beliefs subscales.

There is a suggestive correlation between the Verbal Scale and the Global Beliefs Scale. In a sense, the Verbal Scale reflects a person's ability to acquire knowledge and work with already acquired knowledge, whereas the Performance Scale measures a person's capacity to adapt to new situations (cf. Jackson, 1998). Thus, greater general intellectual capacity appears to be associated with more transcendent beliefs. The correlations of specific IQ subscales with BACARQ subscales provides a more specific interpretation of the data. In particular, there is a strong correlation between Vocabulary and Inner Growth. Vocabulary is a measure of the number of words that a person can use, which itself is thought to reflect a person's interest in knowledge and capacity for greater subtlety of thought (Jackson, 1998). Not surprisingly, es evidenced in Table 3, the Vocabulary Scale is strongly correlated with the personality scale of Understanding, which itself is a measure of a person's curiosity and rational scrutiny of the world (Jackson, 1999). Inner Growth is also correlated with Understanding. The correlations among Vocabulary, Inner Growth, and Understanding suggest that those who are more curious about the world and who have the capacity for greater subtlety of thought are more likely to value inner exploration and self-transformation. 
While the full Performance Scale does not have any statistically significant linear correlations with any of the BACARQ scales, one of the components of Performance, namely Object Assembly, does have statistically significant correlations with the Antiphysicalism and Meaning subscales of the BACARQ. In the Object Assembly task, participants are required to identify an object from a scrambled set of slices of that object (Jackson, 1998). Thus, those better able to see the "whole picture" from a collection of its pieces are those who are more likely to think that there is more to the whole of reality than just the physical world. While the cognitive mechanisms actually involved in a holistic world view may not be the same as those that allow for greater skill in Object Assembly, the correlations of the transcendent subscales with Object Assembly do provide a suggestive analogy for transcendent beliefs.

Quadratic curve fitting and post-hoc analyses did reveal a strong correspondence between the upper half of the Performance Scale scores and all of the BACARQ beliefs scales as seen from Table 5. This suggests that not only are transcendent beliefs associated with greater proficiency in the acquisition and use of acquired knowledge, but also with better psychological abilities that are required to work with novel situations. These abilities include memory, speed of information processing, visualization, perceptual analysis, rapidity of imaginal processing, critical judgment, motivation, and persistence (Jackson, 1998).

Most striking, perhaps, among the results are the correlations between the IQ scales and item Q09 of the BACARQ, indicating unequivocally that those with higher IQ on a number of dimensions of intelligence, including both verbal and performance IQ, are more likely to think that there is more to reality than the physical universe. In fact, fully $23 \%$ of the variance for Q09 can be accounted for by Full Scale IQ. In other words, it is not just that intelligence is correlated with a belief that there is more to reality than the physical universe, but also that the role of IQ in such a transcendent belief is a substantial one.

The question of the relationship of intelligence to paranormal beliefs is addressed by these data to the extent that transcendent beliefs can be considered to be paranormal. However, to further examine this question, the four items of the BACARQ that are perhaps most characteristic of phenomena regarded as paranormal can be formed into a "Paranormal Scale." These are items Q08, Q10, Q16, and Q36 addressing, respectively, out-of-body experiences, extrasensory perception, reincarnation, and life after death. When regarded as a scale, these four items have an alpha value of .55. However, there are no statistically significant correlations between this Paranormal Scale and any of the IQ scales or subscales.

The slight quadratic relationship between intelligence and global beliefs suggests that there may be two different cognitive processes by which transcendent beliefs are held. Those with lower IQ may be reflecting transcendent beliefs that are predominant in popular culture, whereas those with higher IQ may have come to consider transcendent beliefs as more reasonable than materialist beliefs. The existence of such separate cognitive processes may also account for the confusion in the literature concerning the relationship between paranormal beliefs and critical thinking. 
Intelligence Correlates of Transcendent Beliefs 13

It is our hope that others will follow up on the preliminary research presented here by seeking to replicate the results with larger samples, with samples drawn from other populations, and with the use of other measures and investigative techniques. As it stands, however, the results of this study, at least with regard to the population of undergraduate students, are clear: in general, those who are more intelligent are also those with more transcendent beliefs. 
Intelligence Correlates of Transcendent Beliefs 14

\section{References}

Barušs, I. (1990), The personal nature of notions of consciousness: A theoretical and empirical examination of the role of the personal in the understanding of consciousness (Lanham, MD: University Press of America).

Barušs, I. (2000), 'Overview of consciousness research', Informatica: An International Journal of Computing and Informatics, 24 (2), pp. 269-273.

Barušs, I. (2001), 'The art of science: Science of the future in light of alterations of consciousness', Journal of Scientific Exploration, 15 (1), pp. 57-68.

Barušs, I. \& Moore, R. J. (1992), 'Measurement of beliefs about consciousness and reality', Psychological Reports, 71, pp. 59-64.

Barušs, I. \& Moore, R. J. (1998), 'Beliefs about consciousness and reality of participants at 'Tucson II', Journal of Consciousness Studies, 5 (4), pp. 483-496.

Jackson, D. N. (1998), Multidimensional Aptitude Battery-II: Manual (Port Huron, MI: Sigma Assessment Systems).

Jackson, D. N. (1999), Personality Research Form: Manual (London, Ontario, Canada: Research Psychologists Press).

Jewkes, S. \& Barušs, I. (2000), 'Personality correlates of beliefs about consciousness and reality', Advanced Development: A Journal on Adult Giftedness, 9, pp. 91-103.

McCrae, R. R. (1993-94), 'Openness to Experience as a basic dimension of personality', Imagination, Cognition and Personality, 13 (1), pp. 39-55.

Tobacyk, J. \& Milford, G. (1983), 'Belief in paranormal phenomena: Assessment instrument development and implications for personality functioning', Journal of Personality and Social Psychology, 44 (5), pp. 1029-1037.

Roe, C. A. (1999), 'Critical thinking and belief in the paranormal: A re-evaluation', British Journal of Psychology, 90, 85-98. 
Intelligence Correlates of Transcendent Beliefs 15

\section{Author Note}

This paper is based on an undergraduate thesis in psychology written by the first author and supervised by the second. The authors are grateful for research funds made available by King's University College to finance this research. Correspondence concerning this article should be addressed to Imants Barušs at King's University College, 266 Epworth Ave., London, Ontario, Canada N6A 2M3. Electronic mail may be sent to baruss@uwo.ca. 